\title{
Investigating Multi-Array Antenna Signal Convergence using Wavelet Transform and Krylov Sequence
}

\author{
MUHAMMAD AHMED SIKANDER*, RASHID HUSSAIN**, AND ABDUL RAHMAN MEMON*** \\ RECEIVED ON 19.04.2017 ACCEPTED ON 29.05.2017
}

\begin{abstract}
In the present world, wireless communication is becoming immensely popular for plethora of applications. Technology has been advancing at an accelerated rate leading to make communication reliable. Still, there are issues need to be address to minimize errors in the transmission. This research study expounds on the rapid convergence of the signal. Convergence is considered to be an important aspect in wireless communication. For rapid convergence, two ambiguities should be addressed; Eigenvalue spread and sparse identification or sparsity of the signal. Eigen value spread is defining as the ratio of minimum to maximum Eigenvalue, whereas sparsity is defining as the loosely bounded system. In this research, two of these attributes are investigated for MAA(Multi-Array Antenna) signal using the cascading of Wavelet and Krylov processes. Specifically, the MAA signal is applied in the research because nowadays there are many physical hindrances in the communication path. These hurdles weaken the signal strength which in turn effects the quality of the reception. WT (Wavelet Transform) is used to address the Eigenvalue problem and the Krylov sequence is used to attempt the sparse identification of the MAA signal. The results show that the convergence of the MMA signal is improved by applying Wavelet transform and Krylov Subspace.
\end{abstract}

Key Words: Convergence, Wavelet, Krylov Subspace, Transformation, Eigenvalue, Sparse Identification.

\section{INTRODUCTION}

Ie cellular communication, the received signal is a combination of inequalities which are created by the environmental conditions and behavior of the signal due to varying obstacles in the communication path. These phenomena are reflected in the received signal as errors. The idea behind the research is the importance of smooth and fast communication and it is only possible with rapid and efficient convergence.
The Convergence of a signal is of great importance in cellular communication systems and many researchers have addressed the issue by proposing and modifying different Algorithms to minimize MSE (Mean Square Error). Some of them used different WTs like Discrete, Continuous and Multi-resolution to focus on the Eigenvalue and others have tried to identify and control the sparsity in the signalby using Krylov sequence [1-4].

Corresponding Author (E-Mail: rashid.hussain@hamdard.edu.pk)

* Hamdard Institute of Engineering \& Technology, Facultyof Engineering Sciences \& Technology, Hamdard University, Karachi. ** Graduate School, Faculty of Graduate School of Engineering, Sciences \& Information Technology, Karachi.

*** New Ports Institute of Communication \& Economics, Faculty of Engineering \& Technology, New Ports University, Karachi.

Mehran University Research Journal of Engineering \& Technology, Volume 37, No. 1, January, 2018 [p-ISSN: 0254-7821, e-ISSN: 2413-7219] 
Some of the recent research in this domain includes; Super directive Beam forming Based on the Krylov Matrix [5], On the dimension of the Krylov subspace in low complexity wireless communications linear receivers [6], Domain-Decomposition Approach to Krylov Subspace Iteration, IEEE Antennas and Wireless Propagation Letters [7] Model order reduction of large-scale state-space models in fusion machines via Krylov methods [8].

In the latest research it is observed that the researchers used different properties of Krylov in order to achieve their goal [5-8].For instance, in [5] researcher used linear combination of Krylov matrix columns for beam forming. In [6] Krylov matrix solvers are used as an application for low complexity communication receivers in relation with eigenvalues and eigenvectors. In [7] Krylov subspace is utilize as domain decomposition technique by treating each linear combination of columns as a separate subdomain. In [8] Krylov subspace is used to reduce the rank of large scale state space models by taking fusion machine as an example. In [9] researcher compare the convergence performance of two algorithms named as TDNLMS (Transform Domain Normalized Least Mean Square) and TDNLMN (Transform Domain Normalized Least Mean M-Estimation).

This latest research is related to our proposed research because it customs the Krylov matrix to mollify their issues [5-8], whereas it caters convergence issue [9].

The review of past research diverges upon one problem at a time; either Eigen value spread or identification of sparsity, but none of the researchers have used both the constraints simultaneously.

In this research investigation, the Multi-Array Antenna signal is first generated and later converted to Wavelet domain to minimize the Eigenvalue spread. Daubechies is chosen as the mother Wavelet because of its shape, pattern and suitability to the signal. Finally, the Krylov sequence is generated in the same domain to identify the sparsity in the signal.

\section{MATERIALS AND METHOD}

As mentioned earlier the signal Eigenvalue can be minimized by converting the signal to Wavelet domain and the Krylov method can be used to address the sparsity [9-10].The materials and methods are illustrated in Fig. 1.through following phases.

\subsection{Multi-Array Antenna/Smart Antenna}

Two types of Smart antennas have been selected: Switch beam and Adaptive array. In Switch beam we have fixed beams in predetermined directions where Adaptive array have changing beam pattern according to changing environmental condition [11-12]. In this research, Adaptive uniform linear array is used as defined in the Equation (1) [11-12].

$x_{n}(t)=\sum_{i=1}^{N} a\left(\theta_{i}\right) S_{i}(t)$

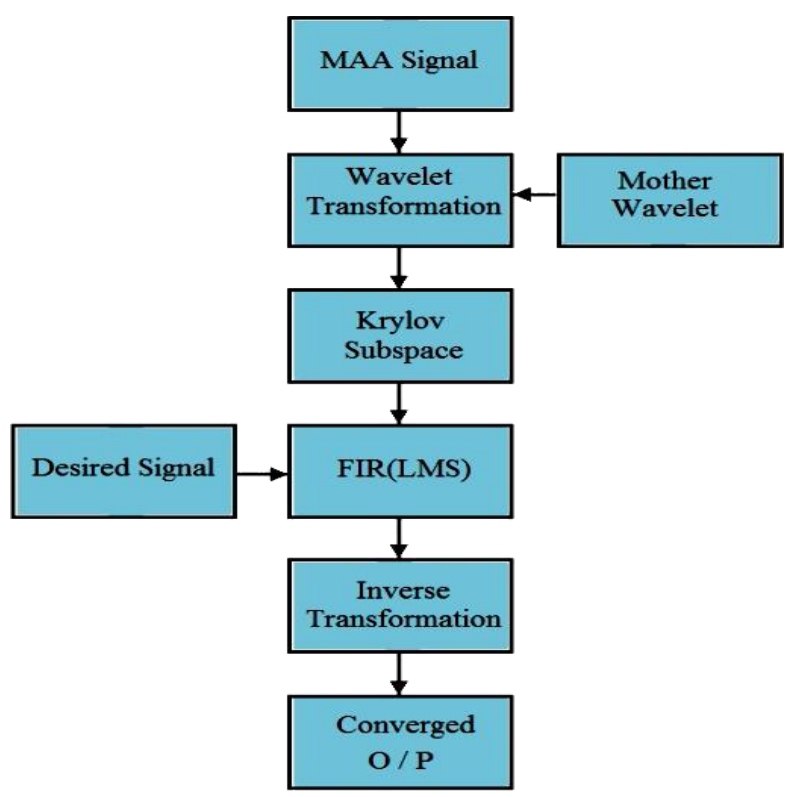

FIG. 1. SCHEMATIC DIAGRAM FOR MULTI-ARRAY ANTENNA SIGNAL CONVERGENCE USING WAVELET TRANSFORM AND KRYLOV SEQUENCE

Mehran University Research Journal of Engineering \& Technology, Volume 37, No. 1, January, 2018 [p-ISSN: 0254-7821, e-ISSN: 2413-7219] 
Here $\mathrm{N}=2$ (no of arrays), $\mathrm{a}\left(\theta_{\mathrm{i}}\right)=\mathrm{e}\left[\mathrm{j}(\mathrm{n}-1) \varphi_{\mathrm{N}}\right]$ (steering vector $), \varphi_{\mathrm{N}}=2 \pi(\mathrm{d} / \lambda) \sin \theta_{\mathrm{N}}, \theta=60^{\circ}$ (Signal arrival angle) assumed $\mathrm{S}(\mathrm{t})$ = single waveform

\subsection{Wavelet Transform}

The WT has a property of defining the position of a signal with its magnitude which gives an edge over the other transforms specially the Fourier Transform. WT decreases the Eigenvalue spread because of its decomposition property $[4,11]$

\subsection{Krylov Subspace}

In linear algebra, the order-r Krylov subspace generated by an $n$ by nmatrix $A$ and a vector $b$ of dimension $n$ is the linear subspace spanned by the values of $b$ under the first $r$ - 1 powers of $A$ (starting from $\mathrm{A}^{0}=\mathrm{I}$ ), that is $[1,10]$

$\mathrm{K}_{\mathrm{r}}(\mathrm{A}, \mathrm{b})=\operatorname{span}\left\{\mathrm{b}, \mathrm{Ab}, \mathrm{A}^{2} \mathrm{~b}, \ldots, \mathrm{A}^{\mathrm{r}-1} \mathrm{~b}\right\}$

In numerical computing, Krylov is the non-stationary type of method which comes under the category of Indirect or Iterative method. The Krylov Subspace method can be used to generate the Krylov sequence in any domain for further processing. The key to improving the convergence is to take all elements of the matrix $A^{r-1}$ which are below a certain threshold value and set them to zero. The consequence is that the sparsity of the matrix increases further, improving convergence times.

\subsection{Finite Impulse Response - Least Mean Square Digital Filter}

There are two types of digital filters defined in the signal processing; FIR (Finite Impulse Response) and IIR (Infinite Impulse Response) [13]. As shown in Fig. 2. FIR is defined as:

- $\quad$ A filter in which the Impulse response settles to zero in a finite duration.

- $\quad$ A feed forward path only. IIR is defined as:
- $\quad$ A filter in which the Impulse response does not settle to zero in a finite duration.

- $\quad$ A filter which contains both the path feed forward and feedback i.e. the present output has not only the input terms but also the output terms.

LMS (FIR) filter is used in the present research to converge the input signal. Reference to the desired signal has been made by minimizing error using mean square error technique and by adjusting the vector weights as mentioned in Equation (3) [13]:

$$
\begin{aligned}
& e(n)=d(n)-u(n) \\
& w(n+1)=w(n)+\mu e(n) x(n)
\end{aligned}
$$

Some attributes that play an important role in the LMS filter includes:

- $\quad$ Help realization of Weiner filter without explicitly solving Weiner-Hopf equation

- $\quad$ Establish stochastic implementation of steepest descent

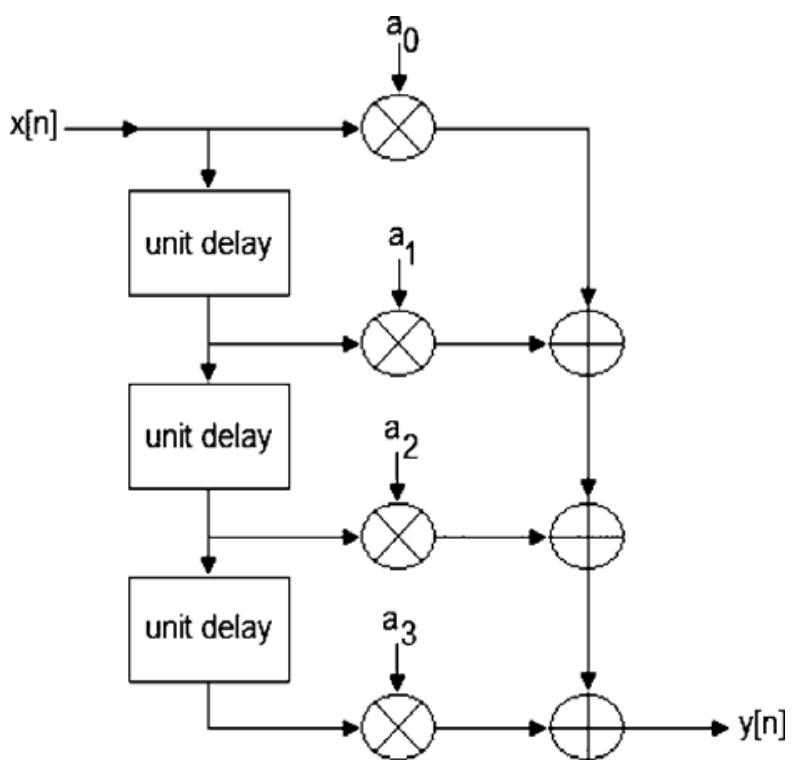

FIG. 2. BLOCK DIAGRAM OF FIR FILTER 
- $\quad$ Remain stable and robust with different signal conditions

- $\quad$ Are easy and simple to implement

\section{ALGORITHM FORMULATION}

This research expounds the efficient converge of the signal in a short span of time (with less number of iterations). Detail block diagram of MAA Signal Convergence using Wavelet Transform and Krylov Sequence Fig. 3.

First of all the MAA signal is generated using [11,12]:

$x_{N}(t)=\sum_{i=1}^{N} a\left(\theta_{i}\right) S_{i}(t)$

$x_{2}(t)=\left[e\left[\left(\left(j(0)\left(2 \pi\left(\frac{d}{\lambda}\right) \sin 45\right)\right)\right] e\left[\left(\left(j(1)\left(2 \pi\left(\frac{d}{\lambda}\right) \sin 45\right)\right)\right][[\sin 45]\right.\right.\right.$

$+\left[e\left[\left(\left(j(0)\left(2 \pi\left(\frac{d}{\lambda}\right) \sin 60\right)\right)\right] e\left[\left(\left(j(1)\left(2 \pi\left(\frac{d}{\lambda}\right) \sin 60\right)\right)\right]\right][\sin 60]\right.\right.$

Here $\mathrm{N}$ is 2 (number of signals), $\mathrm{f}$ is $1 \mathrm{kHz}$ (carrier frequency), $\mathrm{a} \theta_{\mathrm{i}}=\mathrm{e}\left[\varphi(\mathrm{n}-1] \varphi_{\mathrm{i}}\right]$ (Steering vector), $\mathrm{n}=2$ (number of arrays), $\mathrm{S}\left(\mathrm{t}\right.$ ) is signal waveform, $\theta_{1}$ is $45^{\circ}$ (signal arrival angle) assumed, $\theta_{2}$ is $60^{\circ}$ (signal arrival angle), and $\varphi_{\mathrm{i}}$ is $2 \pi(\mathrm{d} / \lambda) \sin \theta_{\mathrm{i}}$.

The required degree of arrival is selected as $60^{\circ}$. The noise to the input signal is simulated using the AWGN (Additive White Gaussian Noise) channel. After AWGN, signal is converted to the Wavelet domain usingSingle level DWT (Approximate Coefficient) [4].

$$
C_{A}=w_{\varphi}\left(j_{o, k}\right)=\frac{1}{\sqrt{M}} \sum_{m} f(x) \varphi_{(j o, k)}(x)
$$

Here $\mathrm{C}_{\mathrm{A}}, \mathrm{W}_{\varphi}$ are Approximate coefficients of DWT, $\mathrm{j}_{\mathrm{o}}$ is Initial value $(0), \mathrm{k}$ is number of points $\left(0,1, \ldots, 2^{\mathrm{j}}-1\right), \mathrm{M}$ is should be power of 2 i.e. $2^{\mathrm{j}}, \varphi_{(\mathrm{jo}, \mathrm{k})}$ is scaling function, $\psi(\mathrm{j}, \mathrm{k})$ is Wavelet function, $\mathrm{n}$ is order of the DWT transform

The DWT gives two forms of the output one is $C_{A}$ approximate coefficients and other is $C_{D}$ detailed coefficients. In DWT $C_{A}$ is used which is the product of the low pass filter. In WT the mother Wavelet is an essential component which has to be selected properly. Therefore, to transform the signal efficiently, and correctly, many trials were conducted and finally Daubechies " $d b 5$ " was selected, as it gave the best output and lowest Eigenvalue, in turn giving the minimized Eigenvalue spread. The next main constraint is the sparsity in the signal and those small value elements in a matrix which should be identified and suppressed before applying to the FIR filter for better convergence rate.

The Krylov sequence is then constructed in the Wavelet domain by estimating Autocorrelation ' $R$ ' matrix and Cross correlation ' $P$ ' vectors using the $\mathrm{C}_{\mathrm{A}}$ coefficients of DWT as in Equation (10) [1,10];

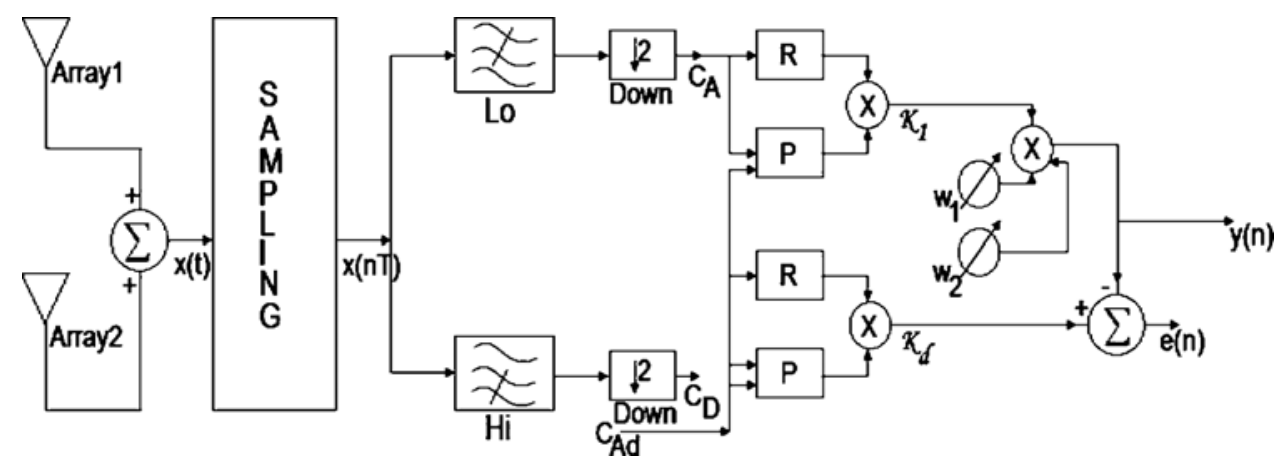

FIG. 3. DETAIL BLOCK DIAGRAM OF MULTI-ARRAY ANTENNA SIGNAL CONVERGENCE USING WAVELET TRANSFORM AND KRYLOV SEQUENCE

Mehran University Research Journal of Engineering \& Technology, Volume 37, No. 1, January, 2018 [p-ISSN: 0254-7821, e-ISSN: 2413-7219] 
$R_{w w}=\sum_{i=0}^{k} w_{\varphi}(k) w(k-i)$

$$
P_{w d}=\sum_{i=0}^{k} w_{\varphi}(k) d(k-i)
$$

The Krylov sequence is generated as the combination of $\mathrm{R}$ and $\mathrm{P}$ given in the Equation (10) $[1,10]$ :

$K(R, P)=\left\{P, R P, R^{2} P, R^{3} P, \ldots, R^{r-1} P\right\}$

The Krylov sequence constructed is a type of subspace which is a linear combination of the terms $R$ and $P$ that makeup a geometric series. By definition it is constructed by ortho-normalizing $R$ matrix and $P$ vectors [10]. Each multiplicative term represents a subspace and the sparse is identified in these subspaces. After that the thresholding method is used to suppress the number of small value elements. This results in the faster convergence by lowering the computation time of the system. The length of $R$ is MxM and length of $\mathrm{P}$ is $1 \mathrm{xM}$ then the length of Krylov subspace will be $2 \mathrm{M}-1$.

After Wavelet and Krylov the major ambiguities are minimized and the input signal is now preconditioned through methods defined above. The signal is then converged using FIR(LMS) filter with reference to desired signal smoothly and efficiently in less duration (number of iterations) as compared to the traditional methods (Wavelet and Krylov separately).

There are several FIR filters available but LMS is chosen due to ease of implementation and robustness.

In many situations for mobile communication there is a prior knowledge of the distribution of the desired signal which is used as a reference signal in FIR filters, e.g. when a mobile consumer is communicating with the server or database for any inquiry, like to manage call services, to load prepaid card, to inquire about prepaid balance or to activate certain service by using different fixed key options.

FIR LMS filter uses the following Equations (11-14) [1314]:

$$
\begin{aligned}
& \mathrm{W}(\mathrm{n}+1)=\mathrm{W}(\mathrm{n})+\mu \mathrm{e}(\mathrm{n}) \mathrm{u}(\mathrm{n}) \\
& \mathrm{u}(\mathrm{n})=\mathrm{k}_{1}^{\mathrm{T}} \\
& \mathrm{e}(\mathrm{n})-\mathrm{K}_{\mathrm{d}}^{\mathrm{T}}-\mathrm{y}(\mathrm{n}) \\
& \mathrm{y}(\mathrm{n})=\mathrm{W}^{*} \mathrm{~K}_{1}^{\mathrm{T}}
\end{aligned}
$$

HereW(n) is adjustable vector weights, $\mu$ is stepsize of LMS filter (between 0.5-0.05), $\mathrm{e}_{(\mathrm{n})}$ is Mean MSE, $\mathrm{n}$ is no of iterations (set to 1500), $\mathrm{K}_{1}$ is Krylov of Input signal, and $K_{d}$ is Krylov of desired signal.

After Convergence, the very next task is to transform the signal to its original domain by means of inverse WT as in Equation (15) [2-4,15],

$$
f(x)=\frac{1}{\sqrt{M}} \sum_{k} y\left(j_{o}, k\right)+\frac{1}{\sqrt{M}} \sum_{j=j_{o}}^{k} \sum_{k} y(j, k)
$$

Here $f(x)$ is, Time domain signal, $y(j, k)$ is Output of LMS in Wavelet domain $\mathrm{M}, \mathrm{J}_{\mathrm{o}}, \mathrm{j}, \mathrm{k}$ are same as in DWT.

\section{RESULTS AND DISCUSSION}

In this section results of the Algorithm are discussed. In order to give better understanding of the proposed algorithm following three scenarios are examined:

- $\quad$ The Convergence of MAA Signal with WT.

- $\quad$ The Convergence of MAA Signal with Krylov sequence.

- $\quad$ The Convergence of MAA Signal with WT and Krylov sequence.

The Configuration used for all scenarios are described in Table 1, Matlab Platform is used to perform the algorithm analysis.

Fig. 4 contains the input signal received at two array antenna with noise and a reference signal used in LMS FIR filter for the signal convergence. 


\subsection{Convergence of MAA Signal with Wavelet Transform}

In this scenario MAA signal is transformed to DWT first before convergence with respect to reference signal using FIR(LMS) filter.

Fig. 5 MSE curve is shown on semi-logarithm scale which is drawn by applying LMS FIR filter directlyto the Wavelet transformed MAA signal.

It is observed that the Error curve is almost constant and there is no decrease in the curve indicating that DWT signal does not converge efficiently, indicating ineffectiveness of LMS filter applied directly to MAA WT signal.

\subsection{Convergence of MAA Signal with Krylov}

In this section MAA signal is used to generate Krylov sequence by finding the autocorrelation matrix $\mathrm{R}$ and cross correlation vector $\mathrm{P}$ and then it is converged with respect to reference signal using LMS FIR filter.

Fig. 6 MSE curve is shown on semi-logarithm scale which is drawn by applying LMS FIR filter directly to the Krylov sequence.

It is observed that the curve is almost constant and at maximum value indicating that the signal is not converging at all. The reason behind is the Krylov subspace for MAA signal which shows that the Krylov sequence does not generate correctly without any proper domain indicating that the proper domain is needed which deal with the frequency coefficients included in the MAA signal as required.

In this scenario the convergence of MAA signal with WT and Krylov sequence is discussed.

Fig. 7 semi-logarithm error curve is shown; it seems obvious that the graph is expeditiously converging toward desired or reference signal, indicating that the WT and Krylov sequence is much more effective in MAA signal convergence with same specifications.

\subsection{Convergence of MAA Signal with Wavelet Transform and Krylov Sequence}

Fig. 8 describes the output of the proposed algorithm that is the converge signal. It contains two graphs one is the converge signal without the inverse wavelet and other is the output taken after the reckoning of inverse wavelet in order to transform the signal to its original domain.
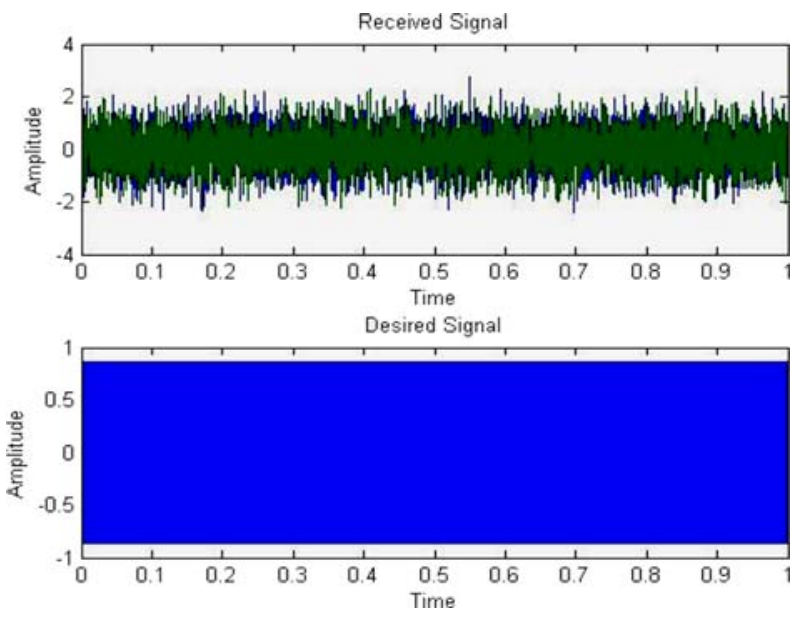

FIG. 4. INPUT SIGNAL

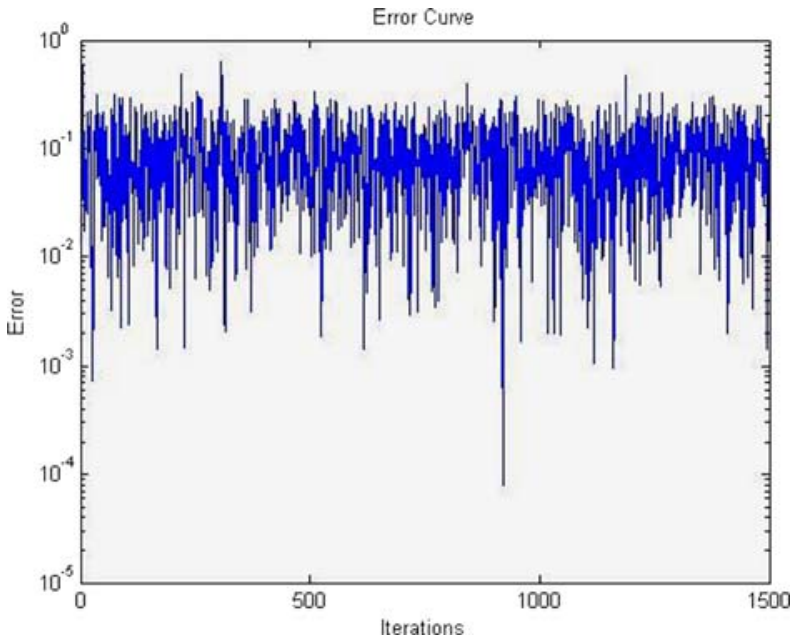

FIG. 5. ERROR CURVE FOR WAVELET TRANSFORM

TABLE 1. CONFIGURATION SCENARIOS

\begin{tabular}{|c|c|c|c|c|c|}
\hline Frequency & $1 \mathrm{KHz}$ & Sampling & $3 \mathrm{KHz}$ & Antenna Arrays & 2 \\
\hline No. of signals & 2 & Waveform & sine wave & Filter Tap & 2 \\
\hline No. of Iterations & 1500 & Step size & $0.5-0.005$ & AWGN & $5 \mathrm{~dB}$ \\
\hline
\end{tabular}

Mehran University Research Journal of Engineering \& Technology, Volume 37, No. 1, January, 2018 [p-ISSN: 0254-7821, e-ISSN: 2413-7219] 


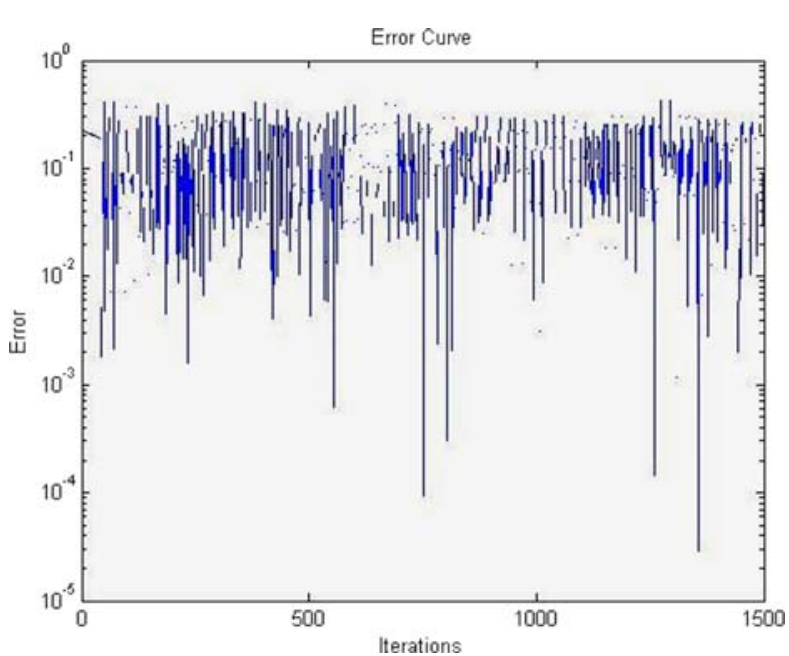

FIG. 6. ERROR CURVE FOR KRYLOV SUBSPACE

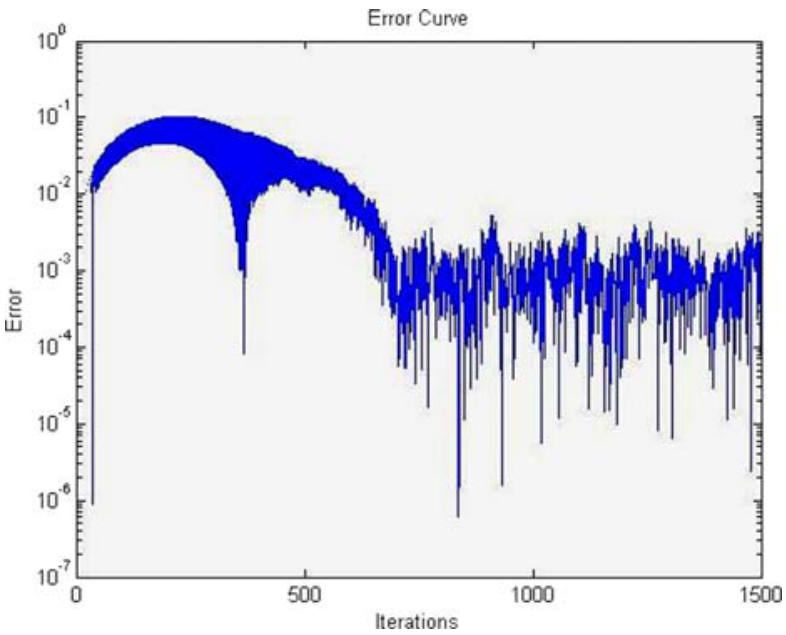

FIG. 7. ERROR CURVE FOR WAVELET TRANSFORM AND KRYLOV SEQUENCE
As observed from Fig. 8 the smooth curve shows an effective convergence proving that the Krylov sequence generation is much more perfect and correct with wavelet domain given the minimum error curve and signal output.

The output is clearly showing that wavelet and Krylov is the right choice and it indicates that their cascading works perfectly for MAA signal.

The comparative study of all the procedures mentioned above had justified that the WT and Krylov sequence has superiority in minimizing the Eigenvalue spread and it deals with sparsity identification better as compared to the other traditional algorithms shown in Table 2.
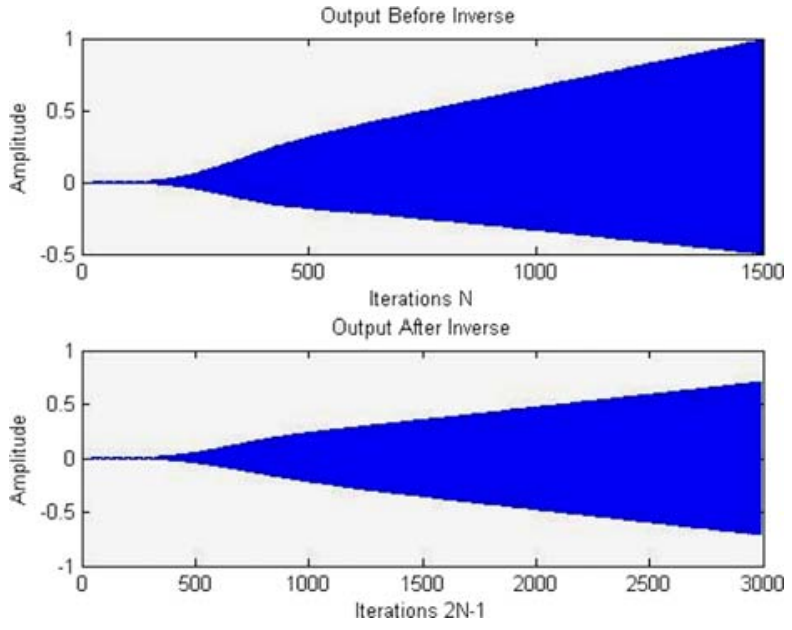

FIG. 8. LMS OUTPUT FOR WAVELET TRANSFORM AND KRYLOV SEQUENCE

TABLE 2. RESEARCH OUTCOME OF MULTI-ARRAY ANTENNA SIGNAL CONVERGENCE USING WAVELET TRANSFORM AND KRYLOV SEQUENCE

\begin{tabular}{|c|c|c|c|c|c|c|c|}
\hline Algorithm & $\begin{array}{c}\text { AWGN } \\
(\mathrm{dB})\end{array}$ & $\begin{array}{c}\text { Time Lapse } \\
\text { (Seconds) }\end{array}$ & Sparsity & MMSE & $\begin{array}{c}\text { Eigen } \\
\text { Value spread }\end{array}$ & $\begin{array}{l}\text { Decrease in } \\
\text { Error Signal }\end{array}$ & Output signal Recognizable \\
\hline \multirow{3}{*}{ WK } & 5 & 71 & 0.018 & $5 \times 10-4$ & \multirow{3}{*}{$\sim 1$} & \multirow{3}{*}{ Yes } & \multirow{3}{*}{$\begin{array}{l}\text { O/P Signal is } \\
\text { recognizable as } \\
\text { per Reference signa }\end{array}$} \\
\hline & $10 \mathrm{~b}$ & 72 & 0.015 & $2 \times 10-4$ & & & \\
\hline & 20 & 80 & 0.015 & $2.4 \times 10-4$ & & & \\
\hline \multirow{3}{*}{ Wavelet } & 5 & 1 & 0 & 0.122 & \multirow{3}{*}{5.116} & \multirow{6}{*}{ No } & \multirow{6}{*}{$\begin{array}{l}\text { O/P Signal is } \\
\text { unrecognizable and far } \\
\text { away from the } \\
\text { reference signal }\end{array}$} \\
\hline & 10 & 1 & 0 & $3.9 \times 10-2$ & & & \\
\hline & 20 & 1 & 0 & $2.9 \times 10-3$ & & & \\
\hline \multirow{3}{*}{ Krylov } & 5 & 1 & 0.047 & 0.359 & \multirow{3}{*}{2.67} & & \\
\hline & 10 & 1 & 0.053 & 0.322 & & & \\
\hline & 20 & 1 & 0.045 & 0.313 & & & \\
\hline
\end{tabular}

Mehran University Research Journal of Engineering \& Technology, Volume 37, No. 1, January, 2018 [p-ISSN: 0254-7821, e-ISSN: 2413-7219] 


\section{CONCLUSIONS}

The reason for traditional algorithm not being effective is the signal property and frequency components of MAA signal. In case of Wavelet, DWT is only controlling Eigenvalue spread up to certain extent, after which it is out of focus. As in Krylov if it used standalone, it does not work on specific frequency band which implies no preconditioned filtration or process and hence includes higher as well as lower frequency components which are not initially control by any proper domain.

Our research has proved especially efficacious in the realm of MAA signal analysis with WT and Krylov sequence and proves that the Eigenvalue spread is minimized and controlled as the signal goes through DWT. And also proves that the Krylov sequence has played an important role by identifying sparsity with certain values which are further modified by thresholding. The convergence is said to be rapid in contrast with other traditional algorithms.

\section{ACKNOWLEDGEMENT}

Authors would like to thank Tong, J., Schreier, P.J., and Weller, S.R., for their research entitled "Design and Analysis of Large MIMO Systems with Krylov Subspace Receivers”. Authors would also like to appreciate Alexei Krylov for providing mathematical basis for Krylov Subspaces, Mallat, S.G., for providing theoretical basis for Wavelet and Mathwork for providing vast computational resources on wavelet transforms and signal processing and to the Graduate School of Engineering Sciences and Information Technology, Hamdard University, Karachi, Pakistan, for their logistic support and services.

\section{REFERENCES}

[1] Tong, J., Schreier, P.J., and Weller,S.R.,’Design and Analysis of Large MIMO Systems with Krylov Subspace Receivers”, IEEE Transactions on Signal Processing, Volume 60, No.5, pp. 2482-2493, 2012.

[2] Chang, S.H., and Chang, C.C.,"The Application of Wavelet-Based Least Mean Square Algorithm in Adaptive Beamforming”, Journal of Marine Science and Technology, Volume 5, No. 1, pp. 13-21, 1997.
[3]

[4] Intelligence, Volume 11, No. 7, pp. 674-693, 1987.

$[5]$

González, R.C., and Woods, R.E., "Digital Image Processing”, Prentice Hall, USA.

Mallat, S.G.,”A Theory for MultiresolutionSignal Decomposition: The Wavelet Representation”, IEEE Transactions on Pattern Analysis and Machine

Huang, G., Jacob, B.J., and Chen, J.," Superdirective Beam Forming Based on the Krylov Matrix", IEEE/ACM Transactions on Audio, Speech, and Language Processing, Volume 24, No. 12 pp. 2531-2543, 2016.

Carvajal, R., and Aguero, J.C., "On the Dimension of the Krylov Subspace in Low Complexity wireless Communications Linear Receivers”, IEEE Latin America Transactions, Volume 14, No. 3, pp. 1192-1198, 2016.

Iupikov, O.A., Craeye, C., Maaskant, R., and Ivashina, M.V., "Domain-Decomposition Approach to Krylov Subspace Iteration”, IEEE Antennas and Wireless Propagation Letters, Volume 15, pp. 1414-1417, 2016.

Bonotto, M., Bettini, P., and Cenedese, A., "Model Order Reduction of Large-Scale State-Space Models in Fusion Machines via Krylov Methods”, IEEE Transactions on Magnetics, Volume 99, pp. 1-1, 2017.

Chan, S.C., and Zhou, Y., "On the Performance Analysis of a Class of Transform-Domain NLMS Algorithms with Gaussian Inputs and Mixture Gaussian Additive Noise Environment”, Journal of Signal Processing Systems, Volume 64, No. 3, pp. 429-445, 2014.

Zhang, Y., Yu, Q., Wu, L., and Huang, P., "A New Transform Domain LMS Algorithm”, IEEE International Conference for Information and Automation, pp. 1256-1259, 2010.

1] Rani, C.S., Subbaiah, P.V., Reddy, K.C., and Rani, S.S., "LMS and RLS Algorithms for Smart Antennas in a WCDMA Mobile Communication Environment”, ARPN Journal of Engineering \& Applied Sciences, Volume 4, No. 6, pp. 78-88, 2009.

Rani, S., Subbaiah, P.V., and Reddy, K.C.,"Music and LMS Algorithms for a Smart Antenna System", IET-UK International Conference on Information and Communication Technology in Electrical Sciences, pp. 965-969, 2007.

Haykin, S., and Kailath, T., “Adaptive Filter Theory Design”, Pearson, USA.

Srar, J.A., Chung, K.S., and Mansour, A.,’Adaptive Array BeamformingUsing a Combined LMS-LMS Algorithm”, IEEE Transactions on Antennas and Propagation, Volume 58, No. 11, pp. 3545-3557, 2010.

Obidin, M.V., and Serebrovski, A.P., "Signal Denoising with the Use of the Wavelet Transform and the Kalman Filter”, Journal of Communications Technology \& Electronics, Volume 59, No. 12, pp. 1440-1445, 2014. 